\title{
Circuit
}

Musiques contemporaines

\section{Fleurs recyclées}

Sur les traces de relations souterraines dans l'Officium breve in memoriam Andreae Szervánszky opus 28 pour quatuor à cordes de György Kurtág ${ }^{1}$

\section{Flowers recycled}

\section{motivic relationships in Officium breve in memoriam Andreae Szervánszky 0p. 28 for string quartet, by György Kurtág}

\section{Friedemann Sallis}

Volume 18, numéro 1, 2008

La fabrique des oeuvres

URI : https://id.erudit.org/iderudit/017908ar

DOI : https://doi.org/10.7202/017908ar

Aller au sommaire du numéro

Éditeur(s)

Les Presses de l'Université de Montréal

ISSN

1183-1693 (imprimé)

1488-9692 (numérique)

Découvrir la revue

Citer cet article

Sallis, F. (2008). Fleurs recyclées : sur les traces de relations souterraines dans l'Officium breve in memoriam Andreae Szervánszky opus 28 pour quatuor à cordes de György Kurtág ${ }^{1}$. Circuit, 18(1), 45-58.

https://doi.org/10.7202/017908ar
Résumé de l'article

Cet article explore une face cachée de l’oeuvre publiée de György Kurtág. Le " motif de la fleur ", d'abord apparu dans Bornemisza Péter mondásai [Dits de Péter Bornemisza], opus 7 (1963-68), a proliféré dans une part significative des composition de Kurtág. L'impact de ce type de relation motivique est mis en lumière à travers l' Officium breve in memoriam Andreae Szervánszky, opus 28 (1988-89), pour quatuor à cordes. La recherche présentée ici est le fruit de la connaissance profonde qu'a l'auteur des documents de travail du compositeur, conservés dans la Collection György Kurtág de la Fondation Paul Sacher. d'utilisation que vous pouvez consulter en ligne. 


\title{
COMPRENDRE LES PROCESSUS COMPOSITIONNELS
}

Fleurs recyclées

\author{
Sur les traces de relations souterraines dans \\ l'Officium breve in memoriam Andreæ Szervánszky \\ opus 28 pour quatuor à cordes de György Kurtág ${ }^{1}$ \\ Friedemann Sallis
}

Nor need any Man be asham'd to be sometimes a Plagiary,

'tis what the greatest Painters, and Poets have allowed themselves...

indeed 'tis hard that a Man having had a good Thought

should have a Patent for it for Ever.

Jonathan Richardson ${ }^{2}$

Ein Dialog ist eine Kette oder ein Kranz von Fragmenten.

Ein Briefwechsel ist ein Dialog in vergrößertem Maßstabe, und Memoralbilien sind ein System von Fragmenten.

Friedrich Schlegel ${ }^{3}$

Tous les hommes ont un secret attrait pour les ruines.

Ce sentiment tient à la fragilité de notre nature,

à une conformité secrète entre ces monuments détruits

et à la rapidité de notre existence.

Chateaubriand ${ }^{4}$

\section{Pratiques de (re)composition}

De la messe-parodie du xve siècle au collage néoclassique et soi-disant 'post' moderne du Xx $x^{e}$ siècle, la recomposition d'un modèle et la réutilisation de fragments, d'idées ou d'objets musicaux, sont solidement enracinées dans les techniques occidentales de composition de la musique - ainsi que dans les autres arts, y compris notamment la littératures. Avant la fin du

1. Cet article s'inscrit dans un projet de recherche soutenu par le Conseil de recherches en sciences humaines du Canada et par la Faculté d'éducation supérieure et de la recherche de I'Université de Moncton. Je suis reconnaissant à Élise Malinge pour les questions, les critiques et les observations qui ont toutes contribué aux résultats présentés ici. Enfin, je remercie chaleureusement Christiane Parchet pour les corrections qu'elle a apportées à ce texte.

2. Jonathan Richardson, A Discourse on the Dignity, Certainty, Pleasure and Advantage of the Science of the Connoisseur (1719), cité dans Christopher Hogwood, Handel, Londres, Thames and Hudson, 1995, p. 275.

3. Friedrich Schlegel, "AthenäumsFragmente", Kritische Schriften, Wolfdietrich Rasch éd., Munich, Carl Hanser, 1964, p. 33.

4. François René, Vicomte de Chateaubriand, cité dans Thomas McFarland, Romanticism and the Forms of Ruin: Wordsworth, Coleridge and the Modalities of Fragmentation, Princeton, Princeton University Press, 1981, p. 15.

5. Pour un survol récent de ce phénomène en musique, ainsi qu'en art visuel et en littérature, voir Linda Cummins, Debussy and the Fragment, Amsterdam, Rodopi B.V., 2006, p. 21-61. 
6. Carl Dahlhaus, Analyse und Werturteil, Musikpädagogik: Forschung und Lehre Vol. 8, Mayence, Schott, 1970, p. 30-33.

7. Lydia Goehr, The Imaginary Museum of Musical Works. An Essay in the Philosophy of Music, Oxford, Clarendon Press, 2002, p. 181-185.

8. David Beveridge, "Dvořák's "Dumka" and the Concepts of Nationalism and Music Historiography," Journal of Musicological Research, 12, 1993. p. 317-320.

9. Né en 1926 à Lugoj, Roumanie, mais de langue hongroise, Kurtág est considéré comme l'un des plus grands compositeurs européens de la fin du siècle dernier. La majeure partie de sa vie professionnelle s'est déroulée à Budapest où il a poursuivi une brillante carrière de professeur de musique de chambre à l'Académie Franz Liszt jusqu'à sa retraite en 1986. Son œuvre a suscité l'intérêt des critiques en Europe de l'Ouest suite au succès de la création des Poslanija Pokojnoj R. V. Trusovoj [Messages de feu Demoiselle R. V. Troussova] opus 17 pour soprano et orchestre de chambre (1976-80), commande de l'Ensemble InterContemporain qui les a créés à Paris en 1981. Depuis, Kurtág a reçu de nombreux prix (entre autre le prestigieux Ernst von Siemens Musikpreis en 1998 et le Grawemeyer Award en 2006) et un nombre croissant d'études consacrées à son œuvre apparaît chaque année. Cependant, l'œuvre de Kurtág n'a toujours pas reçu l'attention qu'elle mérite en Amérique
XviII siècle, l'utilisation d'un modèle (la citation, la paraphrase, la parodie, etc.) est largement répandue. Le geste est principalement une indication de respect de l'emprunteur pour le modèle (Johann Sébastien Bach pour Antonio Vivaldi par exemple). Il constitue aussi la démonstration d'une adhésion à une tradition, ainsi que l'acquisition d'une technique ${ }^{6}$. L'autocitation ne pose elle non plus aucun problème. Quand Georg Friedrich Händel réutilise un air déjà composé dans un nouvel opéra, il ne s'en cache nullement. Gioachino Rossini pratique sans scrupules cette technique au début du XIX ${ }^{e}$ siècle. Pourtant, à cette même époque, le jugement porté sur l'utilisation d'un modèle change drastiquement? : l'originalité devient une des pierres angulaires de la nouvelle esthétique romantique, et le fait d'emprunter au passé tombe sous le signe de la médiocrité. Imiter un modèle n'est bon que pour des épigones, dépourvus de talent et incapables d'exprimer des sentiments forts, personnels et donc authentiques. Avec ce nouveau paradigme esthétique ainsi que la mise en place progressive du cadre légal concernant le droit d'auteur, la pratique d'imitation devient une chose à cacher et certains emploient les grands moyens pour le faire. Comme l'a montré David Beveridge, Antonín Dvořák a utilisé le Quintette pour piano et cordes en Mi bémol majeur de Robert Schumann comme modèle pour son propre Quintette en La majeur, opus 81. Le lien de parenté est particulièrement voyant dans les mouvements lents, ce qui aurait poussé Dvořák à ajouter le titre Dumka après avoir composé le mouvement, dans le vain espoir de camoufler la source de son inspiration avec une indication cosmétique. Le titre folklorisant est ici un écran de fumée destiné à cacher un mensonge honteux. ${ }^{8}$

Et pourtant, originalité et héritage sont les deux facettes d'une même pièce : la première ne peut exister que sur fond d'une tradition technique ou esthétique plus ou moins stable, tandis que l'héritage culturel et artistique, s'il n'est pas constamment réinventé, n'est bon que pour le musée. Ainsi, même si les pratiques d'imitation, de réutilisation et de recomposition d'un modèle ont été repoussées à l'intérieur des portes closes de l'atelier privé du compositeur, elles n'en demeurent pas moins un aspect important de la technique de composition, tant pour les débutants que pour les compositeurs d'âge mûr. Il y a fort à parier (mais il est impossible de le prouver) qu'elles soient aussi importantes pour la musique au $\mathrm{xx}^{\mathrm{e}}$ siècle qu'elles l'ont été pour la musique des siècles passés.

L'œuvre de György Kurtág est un bon exemple de l'étendue et de l'importance de cette pratique au $\mathrm{xx}^{\mathrm{e}}$ siècle. ${ }^{9}$ Le fragment musical joue un rôle prépondérant dans son œuvre depuis presque un demi-siècle. Même ses œuvres de grande envergure sont généralement de longues séries de courtes pièces 
(parfois pas plus de quelques secondes chaque), décrites par Péter Szendy comme des «constellations de microcosmes $»^{10}$. La question se pose alors de savoir comment de telles œuvres forment un tout. Bien entendu, une partie importante de l'œuvre de Kurtág est constituée de cycles de mélodies et dans ces cas, le texte apporte une cohérence indispensable (comme dans les Bornemisza Péter mondásai [Dits de Péter Bornemisza] opus 7, sous-titrés Concerto pour soprano et piano; les Poslanija Pokojnoj R. V. Trusovoj; les Kafka-Fragmente opus 24, pour soprano et violon et ...pas à pas nulle part... Poèmes de Samuel Beckett opus 36, pour baryton, trio à cordes et percussion). Mais qu'en est-il des œuvres instrumentales? C'est ici que l'étude de la citation, de l'autocitation, ainsi que de la réutilisation et la recomposition d'un modèle peut nous apporter une perspective indispensable. En effet, l'CEuvre public (ou plutôt publié) de Kurtág peut être vu comme la pointe d'un énorme iceberg consistant en un vaste réseau de pièces, d'idées et de gestes qui ont irrigué la pensée musicale du compositeur depuis presque cinquante ans. Ce réseau, qui constitue une sorte de «fond de commerce» musical et qu'on peut appréhender à travers une étude soigneuse des manuscrits du compositeur, ouvre une fenêtre sur son atelier de travail et nous permet de mieux comprendre son œuvre" ${ }^{11}$.

Le troisième quatuor à cordes de Kurtág, intitulé Officium breve in memoriam Andrece Szervánszky opus 28 (1984-89), constitue un excellent objet pour l'examen de cette question. Durant environ 12 minutes, l'œuvre est constituée de 15 mouvements distincts. La nature fragmentaire de la composition est renforcée par le fait que la majorité des mouvements sont des arrangements ou emprunts de pièces composées précédemment par Endre Szervánszky et Anton Webern, ainsi que par Kurtág lui-même. Comment cet ensemble de courts fragments, assemblés à partir de pièces écrites sur une période de plus de quinze ans, se constitue-t-il en l'œuvre entière et forte qu'est Officium breve?

\section{Les fleurs dans l'œuvre de Kurtág}

Un des titres souvent rencontrés dans l'œuvre de Kurtág est Virág az ember [littéralement «Fleur l'homme», ce qui signifie «L'homme est une fleur»]. À l'origine cette phrase constitue le texte du troisième segment du troisième mouvement (intitulé «Halál» [La Mort]), des Bornemisza Péter mondásai opus 7 pour soprano et piano. Selon Rachel Beckles Willson, ce segment, qu'elle nomme le motif de la fleur, est aussi important pour l'œuvre qu'il est court: une sorte de moment musical réduit à sa plus simple expression (voir fig. 1 ${ }^{12}$. Le motif évoque la fragilité de l'existence humaine qui se trouve au
10. Péter Szendy, "Musique et texte dans l'œuvre de György Kurtág", dans Philippe Albèra éd. Ligeti Kurtág, Contrechamps, $\mathrm{n}^{\circ} 12-13$, Genève, L’Age d'Homme, 1990, p. 266 ; réimprimé dans Philippe Albèra éd. György Kurtág: Entretiens, textes, écrits sur son ouvre, Genève, Contrechamps, 1995, p. 80. Pour une étude plus récente de cet aspect de l'œuvre de Kurtág, voir Juliana Hodkinson, "Aphorisms, fragments and paratactic synthesis: Hölderlin references and compositional style in György Kurtág's ... quasi una fantasia... ", Musik \& Forskning, 29, 2004, p. 25-32.

11. Kurtág a transféré sa collection de manuscrits musicaux à la Fondation Sacher en 1992. Au printemps 1995 , suite à une année sabbatique passée à la Fondation, l'auteur de cet article a reçu le mandat d'établir un inventaire de la dite collection. En 1995, une partie de celle-ci se trouvait dans un ordre préétabli (notamment les esquisses en rapport avec les Bornemisza Péter Mondásai [Les dits de Péter Bornemisza] op. 7) mais une partie importante était aussi dans un désordre relatif, du moins selon notre point de vue. Notre participation à ce projet a pris fin en août 1999. À l'époque, la collection contenait plus de 15000 pages manuscrites en rapport direct avec le travail de composition de Kurtág.

12. Rachel Beckles Willson, György Kurtág: The Sayings of Péter Bornemisza, Op. 7. A 'Concerto' for Soprano and Piano, Aldershot, Ashgate, 2004, p. 120-124. 
13. Ibid., p. 104

14. Une huitième volume pour piano quatre mains et deux pianos est actuellement en préparation. cœur de l'opus 7: l'homme porte en lui une beauté qui se renouvelle naturellement, mais comme les fleurs, il doit se faner et mourir. Évidemment, le contenu musical et extramusical d'un fragment aussi bref qu'énigmatique ne peut être défini avec précision. Pour le compositeur, le motif de la fleur est une sarabande, et la distribution rythmique et métrique du texte suggère en effet l'évocation fragmentaire d'une danse, tandis que Beckles Willson y voit le jet de fleurs sur un cercueil'³. De par sa simplicité et sa brièveté, le motif apparaît comme un geste improvisé, une esquisse capturée sur le vif. Et pourtant, la distribution palindromique des intervalles mélodiques de la ligne vocale indique que la pièce a été soigneusement composée. Comme nous allons le voir, la double nature de cette musique, qui mélange spontanéité de l'esquisse et calcul, sera transmise chaque fois que le compositeur réutilisera le motif dans des œuvres ultérieures.

FIGURE 1. György Kurtág, Bornemisza Péter mondásai [Les dits de Péter Bornemisza] opus 7, III ("Halál" [Mort])/3. Avec l'aimable autorisation de Universal Edition A.G., (C) 1973 Wien/UE 14493

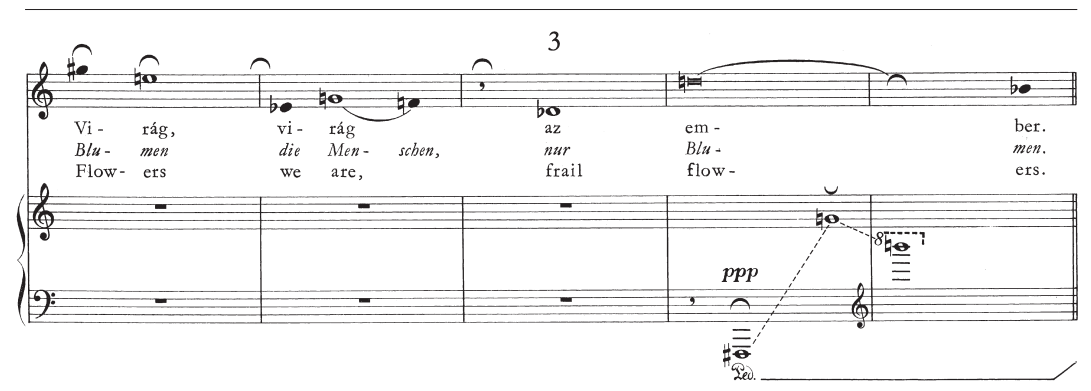

En 1973, Kurtág entreprend la composition de pièces de piano à la demande de la professeure de piano Marianne Teöke. L'année suivante paraît un cahier de petites pièces pour débutants intitulé Eló-Játékok [Pré-jeux]. Ensuite en 1979, Kurtág fait publier quatre recueils intitulés Játékok [Jeux], dont les trois premiers présentent principalement des pièces pour piano solo et le dernier des pièces pour piano quatre mains et deux pianos. Le but initial est pédagogique, et l'ordre des pièces des trois premiers recueils est effectivement gouverné par une progression vers des pièces d'une plus grande difficulté, rappelant les volumes de Mikrokosmos de Béla Bartók. Vingt ans plus tard Kurtág fait publier les volumes 5, 6 et 7 de la série, tous consacrés à des pièces pour piano solo. ${ }^{14}$ Les trois nouveaux volumes portent le sous-titre «Naplójegyzétek, személyes üzenetek» [Entrées de journal intime, messages personnels]. La vocation pédagogique des premiers volumes cède donc le pas 
à des œuvres plus introspectives, explicitement liées aux cercles d'amis, de connaissances et de collègues du compositeur.

Au début de Játékok 1, Kurtág inscrit la partie vocale du motif de la fleur de l'opus 7 comme «Motto » ou devise. ${ }^{15}$ Dans les sept volumes, le titre «Virág az ember » ou des variantes clairement dérivées de ce titre apparaissent 21 fois (dont huit dans le premier volume). Bien sûr, certaines pièces sont plus élaborées que d'autres, mais toutes portent les traces des caractéristiques associées au motif de la fleur mentionnées ci-dessus: brièveté, simplicité, un sentiment de douleur ou de deuil et une reconnaissance implicite de la fragilité de l'existence humaine. Le premier exemple dans Játékok 1, intitulé «Virág az ember...(1a)» (fig. 2), est une parodie du modèle. L'ambiguïté notationnelle concernant la durée dans la version originale augmente considérablement. Et pourtant, la relation entre modèle et variante reste perceptible grâce à la gestique du pianiste et aux paramètres compositionnels suivants:

1. Les nuances - La pièce est marquée pppp avec l'indication verbale que le pianiste doit à peine effleurer les touches de l'instrument;

2. Le registre - Comme dans le modèle, «Virág az ember...(1a)» est organisé en une progression qui va s'élargissant;

3. L'agogique et le phrasé - Le pianiste doit décider quand il faut jouer le prochain événement sonore, ainsi que la durée des silences.

D'après Szendy, «les notes ne comptent pas pour lui, plutôt le geste, plutôt le mouvement $»^{16}$. La simplicité déconcertante de «Virág az ember...(1a)» échappe aux applications pseudo-scientifiques des méthodes analytiques (d'Allen Forte, de David Lewin, etc.), qui ne cherchent qu'à quantifier l'art.

«Virág Nuriának» est daté du 3 juin 1990 (voir fig. 3). Cette pièce fonctionne comme une lettre de condoléances que Kurtág aurait envoyée à

FIGURE 2. György Kurtág, Játékok 1, "Virág az ember... (1a) ", p. 3. Avec l'aimable autorisation de Editio Musica Budapest

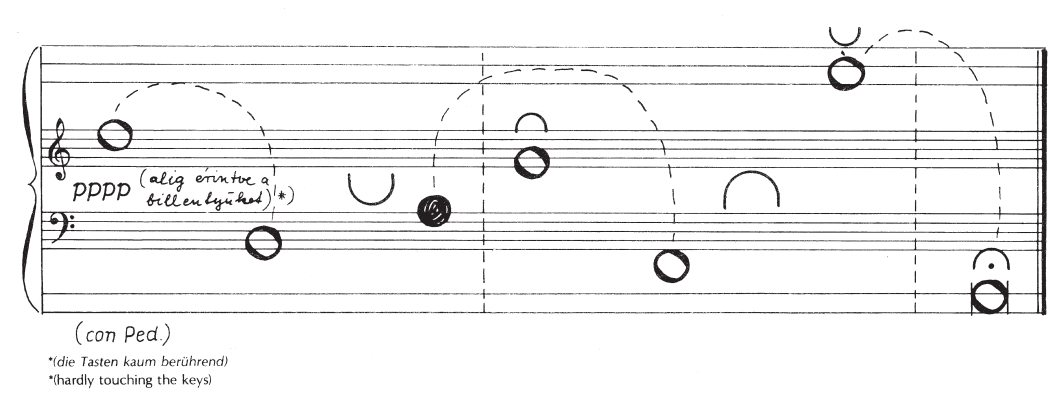

15. György Kurtág, "Táblázat" [Table], Játékok 1, Budapest, Editio Musica, 1979, s. p.

16. D’autres vecteurs présents dans les volumes de Játékok portent des titres tels "Arckép" [Portrait], "Áranyjáték" [Jeu d'ombres] "Verés" [Battements], "Veszekedés" [Se quereller]. Comme "Virág az ember", les autres vecteurs sont constitués d'éléments musicaux et extramusicaux. 
Nuria Schoenberg peu après la mort de son époux, Luigi Nono. Le geste est caractéristique de beaucoup de pièces réunies dans les volumes 5, 6 et 7 (publiés entre 1997 et 2003). Si au départ l'œuvre est un message personnel, le compositeur en a retenu une copie pour lui, et celle-ci fait partie de son œuvre publique. La pièce est clairement calquée sur le motif de la fleur de l'opus 7 , malgré le fait que plus de vingt ans séparent l'écriture des deux compositions. D’un point de vue mélodique, rythmique et notationnel, cette pièce ressemble beaucoup au motif. Comme son modèle, la variante semble être suspendue entre l'apparence du geste spontané et immédiat d'un côté, et le calcul de l'autre: chaque phrase utilise simplement les hauteurs de la gamme de Do majeur plus $\mathrm{Si}$ bémol, et les hauteurs de son de la deuxième phrase sont la rétrograde de la première.

Les volumes de Játékok recèlent 18 autres pièces dont le titre ne contient pas le mot virág, mais dont le contenu musical correspond à des caractéristiques associées au motif de la fleur. La majorité des titres de cette deuxième catégorie contiennent les mots 'emlékére' ou 'in memoriam' et se trouvent

FIGURE 3. György Kurtág, Játékok 6, "Virág Nuriának ", p. 13. Avec l'aimable autorisation de Editio Musica Budapest

a)

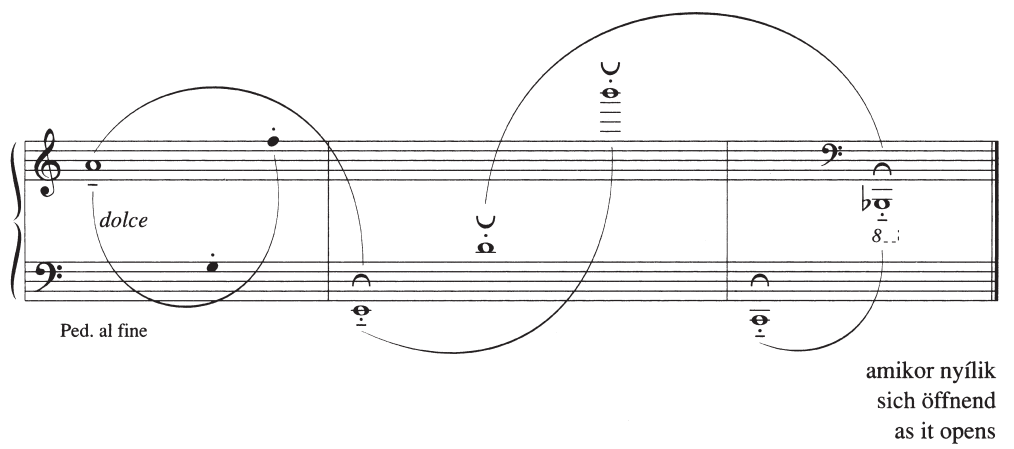

b)

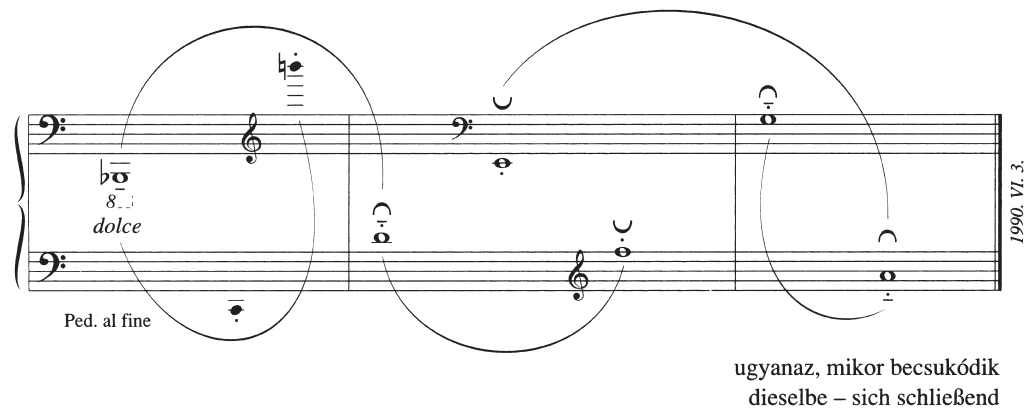
the same as it closes 
dans les volumes 5 à 7 . Comme «Virág Nuriának», plusieurs sont des gestes de condoléances. On trouve également une pièce intitulée «Levél Teöke Marianne-nak» [Lettre à Marianne Teöke] (Játékok 5), ainsi qu’une «Sarabande » pour piano solo (Játékok 1) et une paraphrase de la même pièce pour deux pianos, aussi intitulée «Sarabande» (Játékok 4). Ces deux dernières pièces ont sans aucun doute à voir avec le fait que le compositeur considère le modèle comme une sarabande (voir supra). En tout, les pièces liées au motif de la fleur constituent environ $11 \%$ de toutes les pièces des sept volumes de Játékok. Elles témoignent d'un lien de filiation qu'on pourrait appeler un vecteur esthétique, en ce qu'il dépasse la collection de pièces de piano et traverse l'œuvre entière ${ }^{17}$.

L'inventaire des manuscrits conservés à la Fondation Paul Sacher comprend quatre pièces pour piano solo portant le titre «Virág az ember » ou des variantes dérivées de celui-ci : «Virág az ember » (1984), «Virág az ember... Reismann Mariannenak» [L'homme est une fleur... pour Marianne Reismann] (1980/1982), «Virág helyett» [Au lieu d'une fleur] (1983-1984) et «Virág Körner Éva és Jézus Krisztus születésnapjára » [Fleur pour la fête anniversaire d’Éva Körner et de Jésus Christ] (1984-1985?). Toutes semblent avoir été composées pour les derniers volumes de Játékok, mais, pour des raisons inconnues, elles en ont été écartées. L'inventaire révèle aussi neuf autres pièces pour divers instruments ou voix qui portent le même titre ou des variantes ${ }^{18}$. Enfin, il y aussi des 'fleurs' cachées: de nombreux mouvements ou pièces d'œuvres, publiées ou non, portent également ce titre ou des variantes de celui-ci. Par exemple, la cinquième bagatelle des Bagatellek opus 14/d pour flûte, contrebasse et piano (1979) est intitulée «Virág az ember...» Dans la série Üzenetek [Messages] on trouve aussi une pièce intitulée Inscription on a Grave in Cornwall and Virág az ember... - Kocsis Zoltánnak [Inscription sur une tombe en Cornouailles et L'homme est une fleur... pour Zoltán Kocsis] opus 34d pour chœur mixte et orchestre $(1995)^{19}$. Toutes ces pièces portent des traces du motif de la fleur de l'opus 7, mais la nature du lien, ainsi que le contenu suggéré, sont de plus en plus ramifiés et diffus.

\section{Citation, autocitation, paraphrase et parodie dans officium breve in memoriam Andrea Szervánszky opus 28}

Le troisième quatuor à cordes de Kurtág est un assemblage de fragments autour de deux citations clairement identifiées. D’un côté, le dixième mouvement du quatuor, intitulé «X $[$ Webern Kanon a 4 (Op. 31/VI)] » est une transcription du canon final de la Deuxième Cantate opus 31 d'Anton Webern. De
17. Péter Szendy, "'Es klingt hübsch' sept fragments sur György Kurtág", dans Philippe Albèra éd. György Kurtág: Entretiens, textes, écrits sur son œuvre, Genève, Contrechamps, 1995, p. 187.

18. Friedemann Sallis, Sammlung György Kurtág Musikmanuskripte, Inventare der Paul Sacher Stiftung, 19, Mayence, Schott, sous presse. Une étude sommaire de la base de données contenant de l'information tirée des livres d'esquisses de Kurtág conservés à la Fondation Paul Sacher révèle que le terme 'virág' apparaît 54 fois, soit sur environ 1\% des 5541 pages d'esquisses contenues dans les 106 livres. Friedemann Sallis, Nelson Ouellet, et al., The Sketchbooks of György Kurtág. A database of information extracted from 106 sketchbooks conserved in the György Kurtág Collection of the Paul Sacher Foundation, (C) Friedemann Sallis et l'Université de Moncton, 2004.

19. Le titre Virág az ember Kocsis Zoltának apparaît aussi dans l'inventaire comme une œuvre à part entière, non publiée, écrite en 1995 pour piano, pianino, et percussion. 
20. Pour un survol de la présence de citations et autocitations dans l'œuvre instrumental de Kurtág, voir Rachel Beckles Willson, “Kurtág's Instrumental Music, 1988-1998", Tempo, n²07, décembre 1998, p. 19.

21. Szervánszky aurait découvert l'œuvre de Webern tardivement dans sa carrière, apparemment vers la fin des années 1950. Il a reconnu publiquement sa profonde admiration pour le compositeur dans ses six pièces pour orchestre (1959, créées 1961). Par ailleurs, c'est pendant son séjour à Paris (1957-58) que Kurtág découvre l'importance de Webern et de l'École de Vienne. Comme les Six pièces d'orchestre de Szervánszky, le premier Quatuor à cordes de Kurtág (1959) a été reconnu dès sa création (1961) comme une sorte d'hommage à Webern. György Kurtág, "Werkeinführungen: Officium breve in memoriam Andreæ Szervánszky op. 28 Konzert am 26 Juli Mozarteum ", dans Ligeti und Kurtág in Salzburg, Ulrich Dibelius éd., Zurich, Palladion, 1993. p. 72-73. Pour plus d'information sur ce contexte, voir János Breuer, Negyen év magyar zenekultúrrája [Quarante ans de culture musicale hongroise], Budapest, Zenemäkiadó, 1985; György Kroó, "Bartók and Hungarian Music (1945-1981)", dans Bartók and Kodály Revisited, György Ranki éd., Budapest, Akadémiai Kiadó, 1987, p. 133-146; Friedemann Sallis, "La transformation d'un héritage: Bagatelle $\mathrm{n}^{\circ} 2$ opus 6 de Béla Bartók et Invenció de György Ligeti ", Revue de musicologie 83/2, 1997, p. 281293; Friedemann Sallis, "The Reception of Béla Bartók's Music in Europe after 1945", dans Settling New Scores. Music Manuscripts from the Paul Sacher Foundation, Felix Meyer éd., Mayence, Schott, 1998, p. 255-258; Rachel Beckles Willson, "'Culture is a vast weapon, its artistic force is also strong.' Finding a context for Kurtág's works: an interim report", Contemporary Music Review 20/2-3, 2001, p. 3-37.

22. György Kurtág, Officium breve in memoriam Andreœ Szervánszky Op. 28, l'autre, le dernier mouvement du quatuor présente les douze premières mesures du troisième mouvement, intitulé «Arioso », de la Sérénade pour orchestre à cordes d'Endre Szervánszky. Des quinze mouvements du troisième quatuor à cordes numérotés par le compositeur, seuls quatre ont été composés originalement pour cette œuvre (les mouvements IV, VI, IX et XIV). Les autres mouvements sont des citations, autocitations ou paraphrases d'œuvres écrites auparavant ${ }^{20}$. Le tableau de la figure 4 montre la généalogie immédiate de chaque mouvement du quatuor à cordes.

Le choix de Kurtág de placer la musique de Webern et de Szervánszky au cœur d'Officium breve est lié aux dates de composition des deux œuvres citées (voir fig. 4) : les deux œuvres ont été écrites à l'époque où Kurtág recevait sa formation professionnelle en musique. De plus, elles incarnent les deux legs esthétiques sur lesquels est fondé l'œuvre de maturité de Kurtág: l'héritage hongrois de Bartók et Zoltán Kodály d'un côté, et l'École de Vienne de l'autre. Le troisième quatuor à cordes de Kurtág est donc à la fois un hommage à Szervánszky et un geste de reconnaissance à l'égard de l'impact important, mais non reconnu officiellement, qu'a eu l'œuvre de Webern en Hongrie après l'échec de la Révolution hongroise (novembre 1956) ${ }^{21}$. Officium breve est donc une œuvre profondément autobiographique.

Au-delà des deux citations, Officium breve est constitué surtout d'une série d'arrangements et de paraphrases de pièces que Kurtág a lui-même composées dans les quinze ans précédant le travail sur le quatuor. Dans une page d'introduction à la partition, Kurtág identifie quatre mouvements du quatuor, écrits bien avant qu'il commence à travailler sur l'Officium breve. Chaque pièce avait été composée comme message de condoléances suite aux décès de Tibor Turcsányi (I), Zsolt Baranyai (II), Gabriella Garzó (VIII) et György Szoltsányi (XI)22. Curieusement, Kurtág ne mentionne pas le fait que le mouvement XIII (comme le mouvement VIII) est une paraphrase de la pièce (publiée dans Játékok 5) qu'il a écrite à la mémoire de Gabriella Garzó (voir fig. 1). Donc, cinq autocitations dans l'Officium breve (I, II, VIII, XI et XIII) peuvent être directement liées au vecteur esthétique issu du motif de la fleur de l'opus 7. De plus, deux des mouvements composés originalement pour Officium breve ont aussi un lien de filiation avec ce vecteur. L'étude des esquisses de Kurtág montre que le mouvement VI devait porter le titre «Virág az ember I Hommage à Webern » et le mouvement IX devait porter le titre «Virág az ember II ». Les indications détaillées concernant les nuances, le phrasé et la couleur instrumentale des deux mouvements font allusion au style pointilliste de la musique de Webern. En même temps, la brièveté de chaque mouvement, ainsi que l'utilisation de nuances extrêmement douces 
FIGURE 4. Généalogie de chacun des quinze mouvements d'Officium breve in memoriam Andrea Szervánszky opus 28

I. Largo: autocitation légèrement modifiée de Virág az ember (Turcsányi Tibor emlékére) [L’homme est une fleur (À la mémoire de Tibor Turcsányi)] pour violoncelle, 1978, manuscrit non publié23;

II. Più andante : autocitation arrangée pour quatuor à cordes d'In Memoriam Baranyai Zsolt [À la mémoire de Zsolt Baranyai] pour deux flûtes à bec ou deux hautbois ou deux clarinettes et harpe ou harmonium ou piano ou cymbalum, 1978, manuscrit non publié;

III. Sostenuto, quasi giusto: autocitation arrangée pour alto et violoncelle d'Hommage à Szervánszky Csend a [Silence a] pour piano, 1973-75, publié dans Játékok 3 (1979);

IV. Grave, molto sostenuto: composé originalement pour l'opus 28 (Cependant les trois premières mesures de ce mouvement empruntent la série utilisée par Webern dans le canon du dernier mouvement de sa Cantate opus 31. Donc le mouvement peut être considéré comme une courte parodie de la citation présentée dans le mouvement X.);

V. (Fantasie über die Harmonien des Webern-Kanons) Presto: paraphrase de la structure harmonique du canon du dernier mouvement de la Cantate opus 31 de Webern;

VI. (Canon a 4) Molto agitato : composé originalement pour l'opus 28;

VII. Canon a 2 (frei, nach op. 31/VI von Webern) Sehr fliessend: paraphrase des voix extérieures du canon du dernier mouvement de la Cantate opus 31 de Webern

VIII. Lento : paraphrase de Virág - Garzó Gabinak [Fleur - pour Gabi Garzó] (variante a) pour piano, 1981, publié dans Játékok 5 (1997);

IX. Largo : composé originalement pour l'opus 28;

X. [Webern: Kanon a 4 (op. 31/VI)] Sehr fliessend: citation arrangée pour quatuor à cordes du canon du dernier mouvement de la Cantate opus 31 de Webern, $1942^{24}$ (Le mouvement Xa est une reprise variée de mouvement X. Elle remplace la reprise exacte dans le dernier mouvement de la Cantate de Webern.);

XI. Sostenuto : autocitation arrangée pour quatuor à cordes de Szoltsányi György emlékezete [À la mémoire de György Szoltsányi] pour piano, 1988, publié dans Játékok 5 (1997);

XII. Sostenuto, quasi giusto : autocitation arrangée pour quatuor à cordes d'Hommage à Szervánszky Csend $b$ [Silence b] pour piano quatre mains, 1973-75, publié dans Játékok 3 (1979);

XIII. Sostenuto, con slancio : paraphrase de Virág - Garzó Gabinak [Fleur - pour Gabi Garzó] (variantes c, d, e) pour piano, 1981, publié dans Játékok 5 (1997)

XIV. Disperato, vivo : composé originalement pour l'opus 28;

XV. Arioso interrotto (di Endre Szervánszky): citation arrangée pour quatuor à cordes des douze premières mesures du troisième mouvement, intitulé "Arioso", de la Sérénade pour orchestre à cordes d’Endre Szervánszky, 1947-1948.
23. Tous les manuscrits mentionnés dans cette liste sont conservés dans la Collection György Kurtág de la Fondation Paul Sacher (voir Sallis, Sammlung György Kurtág, op. cit.).

24. Webern a terminé sa deuxième Cantate en 1943, cependant le sixième et dernier mouvement a été composé en 1942 (Anton Webern, Letters to Hildegard Jone and Josef Humplik, Bryn Mawr, Theodore Presser, 1967, p. 47). 
25. Cette structure, dans laquelle des pièces écrites préalablement sont intercalées parmi d'autres, peut rappeler des techniques narratives innovatrices, développées au XIXe siècle par des auteurs tels E.T.A. Hoffmann dans son Kater Murr ou Gérard de Nerval dans son roman Aurélia. Cf.Cummins, Debussy and the Fragment, p. 56-57. et de grands sauts mélodiques, les lient aussi au vecteur du motif de la fleur. Presque la moitié du troisième quatuor (sept mouvements sur quinze: I, II, VI, VIII, IX, XI, XIII) constitue alors une sorte de bouquet de fleurs interfoliées entre des mouvements qui font explicitement référence aux œuvres de Webern et Szervánszky 25 . Le titre même du quatuor à cordes renforce cette interprétation. L'indication latine «in memoriam » suggère un lien entre cette œuvre et de nombreuses pièces qui sont liées au motif de la fleur. Mais comment cet assemblage peut-il constituer un tout?

FIGURE 5A Endre Szervánszky, Sérénade pour orchestre à cordes, III. "Arioso", mesures 1-20. Avec l'aimable autorisation de Editio Musica Budapest
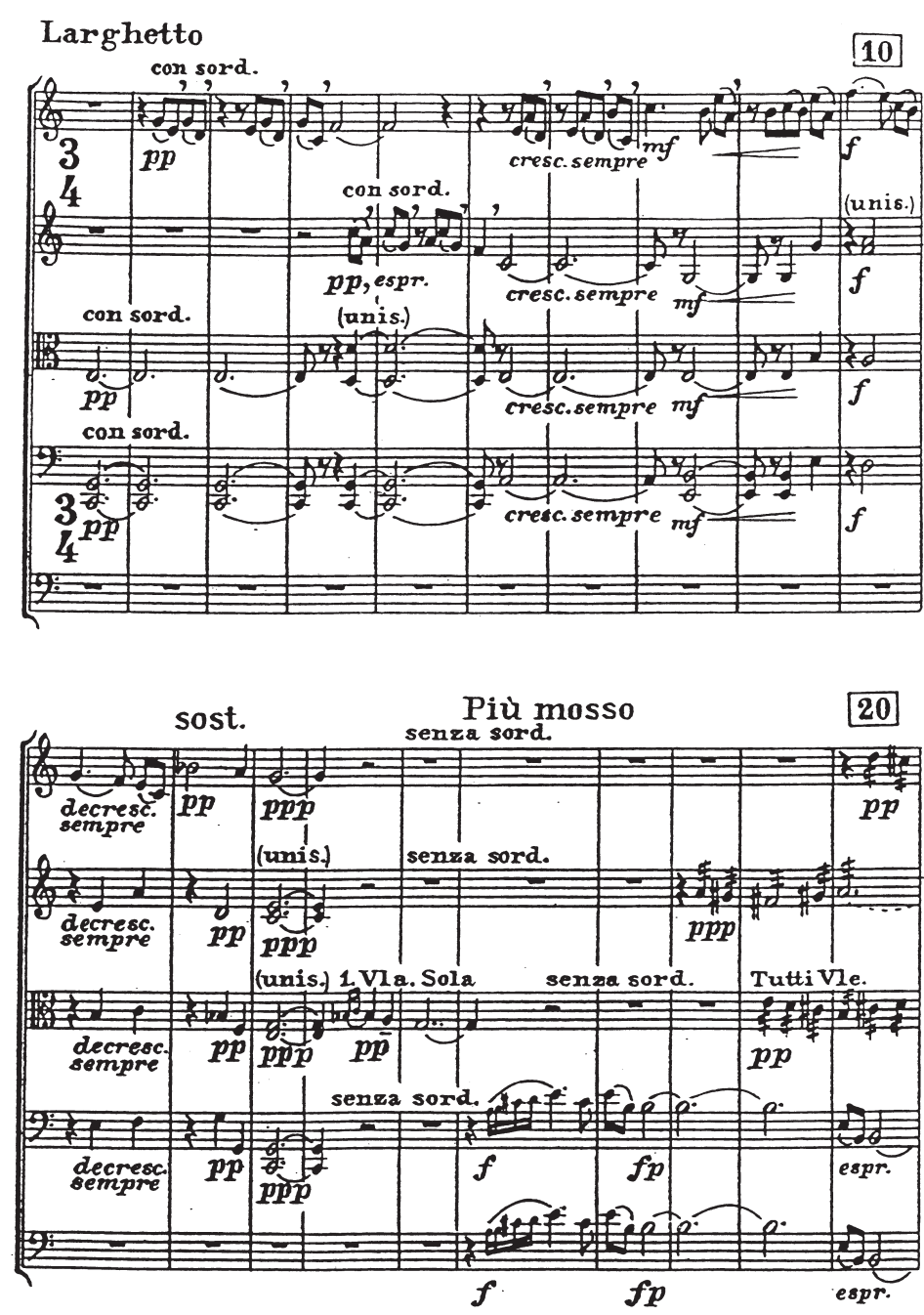
Officium breve est une des compositions de Kurtág qui a reçu le plus de commentaires, de recensions, de revues et d'analyses, et dès le début s'est posée la question de l'unité de l'œuvre ${ }^{26}$. Partant de l'idée que le troisième quatuor à cordes est « une synthèse de techniques de compositions variées, de sources musicales et d'associations extramusicales », Benjamin Frandzel cherche le fondement de cette synthèse dans une étude des structures de hauteurs de $\operatorname{son}^{27}$. Il identifie un «motif de broderie » [the neighbor motif] dérivé à la fois de la citation de Szervánszky et de la citation de Webern, qui serait l'une des deux sources de cohésion structurale de l'œuvre entière (l'autre étant la technique de canon). De plus, il affirme que les douze premières mesures de l'Arioso de Szervánszky ont inspiré la composition des mouvements I, II, III, VIII, IX, XI, XII et XIII puisque le mouvement de broderie figure dans tous ces derniers ${ }^{28}$. Cette affirmation est manifestement fausse puisque la moitié de ceux-ci (les mouvements I, II, VIII et XIII) ont été composés longtemps avant que Kurtág commence son travail sur Officium breve ${ }^{29}$. Alan E. Williams, à la recherche d'une réponse à la même question concernant la cohésion de l'Officium breve, suit une piste différente. Il observe que dans les œuvres de Kurtág, et particulièrement dans Officium breve, «on ne trouve aucune relation stable entre motif musical et association ou idée extramusicale: seulement un réseau d'associations fluides et complètement subjectives. Il n'y a pas de langage kurtágien d'associations musicales susceptibles d'être quantifiées ou cataloguées par l'auditeur »30.

FIGURE 5B György Kurtág, Játékok 3, "Hommage à Szervánszky Csend a [Silence a] ", p. 36. Avec l'aimable autorisation de Editio Musica Budapest

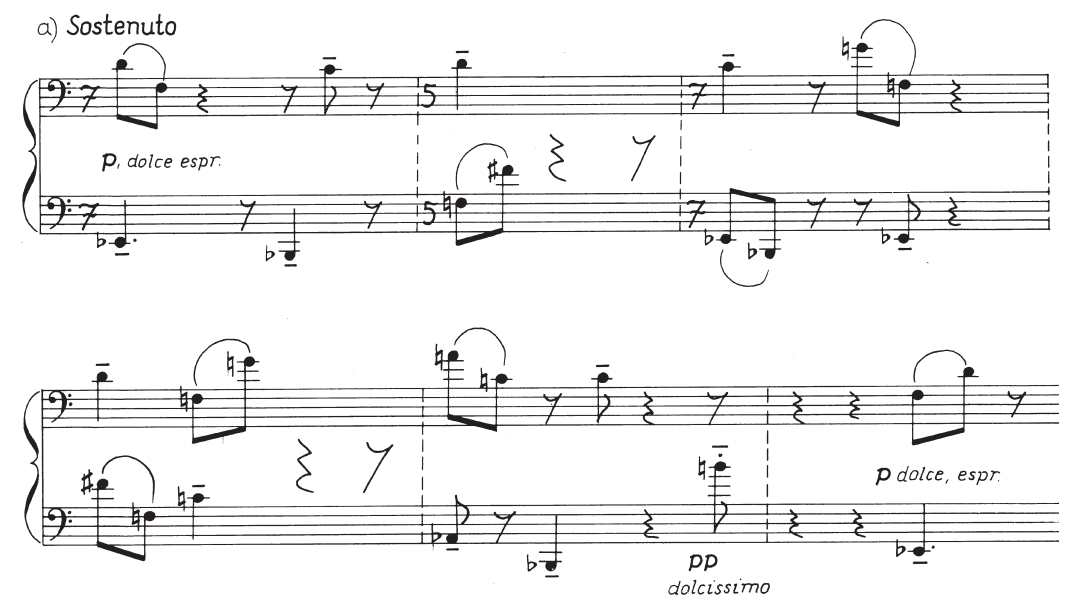

26. Dans un premier commentaire publié peu après la création de l'œuvre, Stephen Walsh observe que la forme d'Officium breve semble émerger graduellement d'un réseau de références (Stephen Walsh, "A brief office for György Kurtág", The Musical Times, $n^{\circ} 130$, Septembre 1989, p. 525526.

27. "György Kurtág's Officium Breve in memoriam Andrea Szervánszky, op. 28 , for string quartet, is a remarkable synthesis of varied compositional techniques, musical sources and extramusical associations" (Benjamin Frandzel, "A Canon Across Time: György Kurtág's Officium Breve in memoriam Andrea Szervánszky, op. 28", Studia Musicologica, 43/3-4, 2002, p. 383).

28. Ibid., p. 385.

29. L'étude analytique de l'Officium breve par Peter Hoffmann souffre de problèmes semblables (Peter Hoffmann, "Post-Webernische Musik? György Kurtág's Webern-Rezeption am Beispiel seines Streichquartetts op. 28", Musiktheorie, $n^{\circ}$ 7, 1992, p. 129-148.

30. "...there is no stable relationship between musical motive and association or extramusical idea, only a network of fluid and totally subjective associations. There is no Kurtágian language of musical associations that the listener may quantify and catalogue" (Alan E. Williams, "Kurtág, Modernity, Modernisms" Contemporary Music Review 20/2-3, 2001, p. 66-67). 
31. Pour une analyse exhaustive de la fonction du silence dans ce mouvement, ainsi que dans l'officium breve, voir Élise Malinge, Tel un Silence. Fonctions du silence et unité formelle dans l'Officium breve in memoriam Andreæ Szervánszky op. 28 de György Kurtág, Mémoire de Maîtrise, Universités de Moncton/Canada et Poitiers/France 2003
Le troisième mouvement présente un excellent exemple de ces multiples associations et correspondances engendrées habituellement par la musique de Kurtág. Une première correspondance peut être établie entre la version originale pour piano solo «Hommage à Szervánszky Csend a [Silence a]» et «l'Arioso» de la Sérénade de Szervánszky (fig. 5a). Les soupirs entrecoupés de silences qui caractérisent les parties de violons 1 et 2 dans "l'Arioso" deviennent la substance même d' "Hommage à Szervánszky Csend a [Silence a] $»^{3^{1}}$ (fig. 5b).

FIGURE 6 György Kurtág, Officium breve in memoriam Andreœ Szervánszky, opus 28, mouvements I, II et III. Avec l'aimable autorisation de Editio Musica Budapest
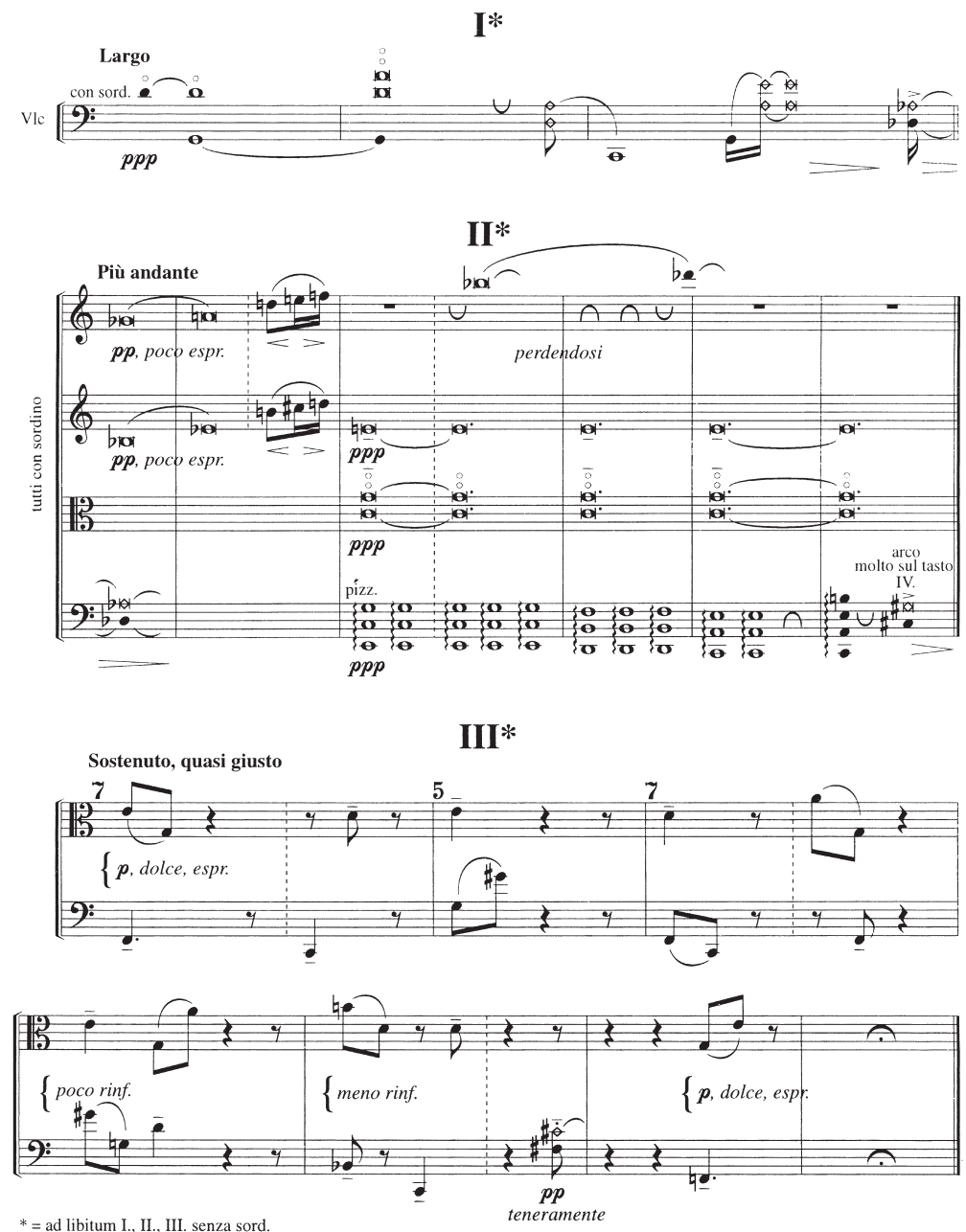
Une autre correspondance concerne l'organisation des hauteurs de son au début de l'Officium breve. Le compositeur a lui-même admis que les deux citations représentaient une division des mouvements de l'œuvre en deux parties, l'une plutôt tonale et l'autre franchement atonale ${ }^{32}$. Les trois premiers mouvements forment un groupe clairement lié à la partie tonale de l'œuvre: tandis que les mouvements I et II tournent autour de Do (et même Do majeur, voir mouvement II, mesure 3), le troisième mouvement répond en soulignant $\mathrm{Fa}$ (voir fig. 6).

Dans son étude du deuxième mouvement, Alan E. Williams note que s'il y a suggestion de tonalité dans l'œuvre de Kurtág, la fonction de celleci est paradoxalement très faible, sinon complètement inexistante33. Le troisième mouvement présente un bon exemple de cette tonalité affaiblie. Premièrement, si on exclut la dernière mesure vide, presque la moitié de la pièce est occupée par le silence (17 croches sur 40). Deuxièmement, la majorité des intervalles harmoniques sont des dissonances non résolues, ce qui enlève tout sens d'une progression et contribue au sentiment de flottement qui envahit le mouvement. Bien qu'écrit pour deux instruments (qui reprennent les deux mains du pianiste d'« Hommage à Szervánszky Csend a [Silence a]»), le mouvement peut être déconstruit sur trois portées montrant une structure contrapuntique (fig. 7).

FIGURE 7 Décomposition du troisième mouvement de l'Officium breve in memoriam Androe Szervánsky
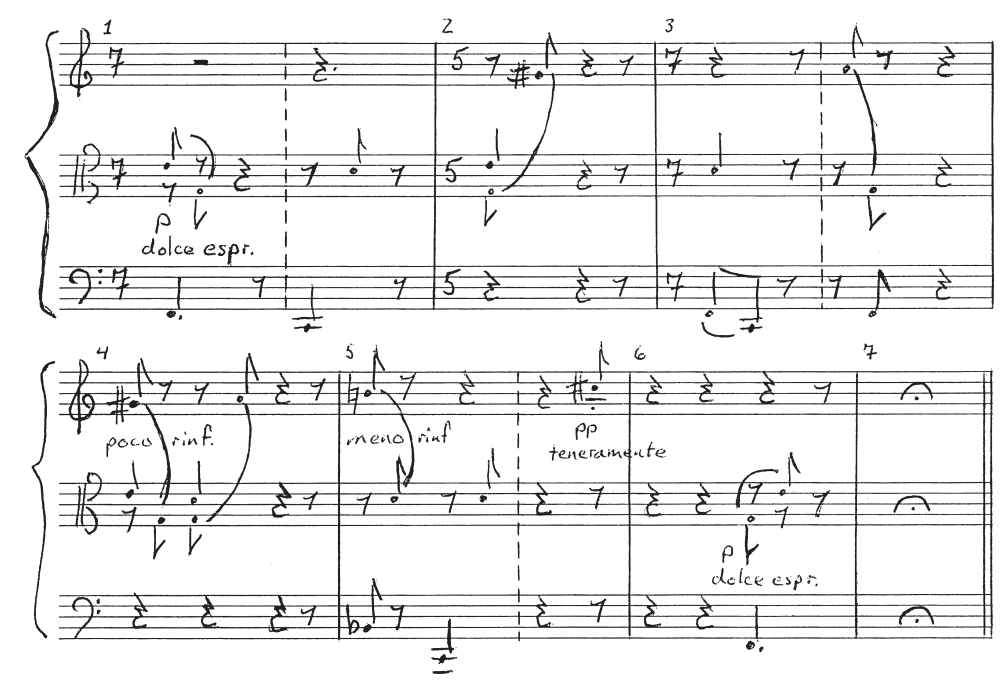

32. György Kurtág, " Mein drittes Streichquartett", manuscrit conservé dans la Collection György Kurtág, Fondation Paul Sacher.

33. Williams, "Kurtág, Modernity, Modernisms", p. 56-57. 
34. Gianmario Borio, Musikalische Avantgarde um 1960 (Laaber, Laaber Verlag, 1993), p. 171.
35. Il cite le cas de l'autocitation d'un fragment de Papillons opus 2 dans "Florestan" du Carnaval opus 9 (Charles Rosen, The Romantic Generation, Cambridge, Harvard University Press, 1995, p. 98-100). Pour une étude approfondie du même phénomène dans la littérature romantique allemande, voir Laurie Ruth Johnson, The Art of Recollection in Jena Romanticism: Memory, History, Fiction and Fragmentation in Texts by Friedrich Schlegel and Novalis, Tübingen, Max Niemeyer Verlag, 2002.

36. Cummins, Debussy and the Fragment, p. 52-53. Cummins tire sa lecture des fragments de Schlegel de Peter Firchow, "Introduction", dans Friedrich Schlegel's "Lucinde" and the Fragments (Peter Firchow, éd. et trad.), Minneapolis, University of Minnesota Press, 1971, p. 18.
La voix basse souligne clairement les degrés I, IV et $\mathrm{V}$ en Fa. Au milieu, un Sol répété est doublé d'une alternance répétée entre Do et Ré. Finalement, la voix du haut dessine une ligne montante de Sol dièse à Do dièse. Le mouvement exclusivement conjoint des deux portées supérieures constitue une compensation indirecte pour les dissonances harmoniques non-résolues qui résonnent du début à la fin du mouvement. Au lieu de présenter une forme de tonalité reconstruite, les sons et le silence du troisième mouvement évoquent le souvenir de ce qu'était la tonalité. Rien n'est affirmé. Au contraire, comme l'écrit Gianmario Borio, l'auditeur doit reconstituer le sens de l'œuvre à partir des suggestions et allusions laissées par le compositeur ${ }^{34}$. À la place d'une architecture tonale, nous sommes confrontés aux débris d'un édifice abandonné et en ruines. Le silence du troisième mouvement est hanté par l'idée de plénitude que la tonalité offrait autrefois aux compositeurs de talent.

Dans son examen de la citation et de l'autocitation dans l'œuvre de Robert Schumann, Charles Rosen note qu'une citation est un souvenir rendu public. De plus il suggère que les techniques de citation, d'autocitation, de paraphrase et de parodie constituent une métaphore de la mémoire et de son fonctionnement. Comme un souvenir éphémère, celles-ci préservent des fragments du passé en même temps qu'elles les transforment et parfois les déforment35. En tant que telles, ces techniques jouent deux rôles qui semblent diamétralement opposés. D'un côté elles déstabilisent l'œuvre dans laquelle elles sont insérées, puisqu'elles renvoient l'auditeur à l'extérieur de l'œuvre en l'obligeant à réfléchir sur les contextes musicaux et extramusicaux. De l'autre, elles renforcent la cohésion parce qu'elles invitent l'auditeur à chercher une unité à un autre niveau de cohérence. Linda Cummins observe que les Athenäums-Fragmente de Friedrich Schlegel rappellent de fausses ruines qu'on construisait au XVIII ${ }^{\mathrm{e}}$ siècle : leur fonction est d'inciter le spectateur à construire mentalement quelque chose qui n'a jamais vraiment existé3 ${ }^{6}$. Dans Officium breve, l'auditeur est confronté à une situation analogue. L'œuvre lui présente un réseau d'idées musicales, saturé de références et d'associations extramusicales, dans lequel l'interprète (qu'il soit musicien, analyste ou auditeur) doit induire un sens. L'unité de l'œuvre ne peut être trouvée seulement dans une analyse technique du matériau sonore, mais aussi dans la contemplation du silence qui l'habite et l'entoure. 